\title{
Systematic X-ray study of GeV gamma-ray emitting radio galaxies
}

\section{Hiroto Matake ${ }^{a, *}$ and Yasushi Fukazawa ${ }^{a, b}$}

${ }^{a}$ Hiroshima University, Department of Physical Sciences, Hiroshima prefecture 739-8526, Japan

E-mail: matake@astro.hiroshima-u.ac.jp, fukazawa@astro.hiroshima-u.ac.jp

\begin{abstract}
Only 10\% of active galactic nuclei (AGNs) have a powerful radio jet, and these objects are roughly classified either radio galaxy or blazar. Blazars are bright but its radiation almost comes from the core jet because of strong beaming effect, while radio galaxies whose beaming effect is weak show various emission components from outer-layer jet, outer jet, and disk/corona. Therefore, radio galaxies are considered to be important objects to understand a relation between jet and disk. To study this relation is considered to be an important point to understand jet ejection mechanism X-ray emission from radio galaxies contain both jet and disk/corona radiation. Thus, we have to investigate contributions from jet and disk/corona to the X-ray band.

In this work, we investigate $\mathrm{X}$-ray and Gamma-ray properties of $\mathrm{GeV}$ emitting radio galaxies listed in the 4FGL-DR2 catalog. We use X-ray data of Swift/XRT. We studied time variability, the relation between X-ray and gamma-ray photon index, together with accre-tion rate, and found they are classified into 3 groups; X-ray emission in the first group is dominated by jet emission, X-ray of the second group is dominated by disk/corona emis-sion, and for the third group both jet and disk/corona contribute to the X-ray band.
\end{abstract}

$37^{\text {th }}$ International Cosmic Ray Conference (ICRC 2021)

July 12th - 23rd, 2021

Online - Berlin, Germany

\footnotetext{
*Presenter
} 


\section{Introduction}

Black Holes (BHs) at the center of galaxies have a $10^{6}-10^{9}$ solar mass and thus are called Supermassive Black hole (SMBH). When a large amount of matter accretes onto SMBH, the accreting matter shines brightly. This phenomenon is called active galactic nuclei (AGN).

AGN has many subclasses and there are two main types; one is radio-loud (RL) AGN and the other is radio-quiet (RQ) AGN. A fraction of RL-AGNs is about $10 \%$, and it has jet radiation from pc-scale region. RL-AGNs are furthermore classified with respect to the viewing angle as blazar if it is a few degrees and classified as radio galaxy (RG) or misaligned AGN (MAGN) if it is typically more than 10 degree. Therefore, jet emission from blazar is affected by so called "beaming effect" the observed emission is dominated by beamed jet. On the other hand, RG has less beaming effect than blazar and we can observe various radiation components from faint part of jet, lobes, accretion disk, corona, and parent galaxy.

In the Fermi/LAT observation the number of detections of $\mathrm{GeV}$ gamma-ray is drastically increased. Their Spectral energy distribution (SED) is well fitted SSC model which explain 2 peaks on the SED; synchrotron radiation and Synchrotron Self-Compton (SSC) radiation. SSC radiation distributes over $\mathrm{X}$-ray to gamma-ray, thus we need to understand jet radiation in $\mathrm{X}$-ray and gamma-ray in order to understand SED of GeV RG.

It is difficult to distinguish disk/corona radiation from jet radiation because both are observed in the X-ray band. In previous systematic study about X-ray radiation of RG to distinguish the origin (Fukazawa et al. 2014[1]), not only X-ray spectral index but also X-ray variability, Fe-K $\alpha$ line, and [OIII] line luminosity was investigated. They indicated that, there is a trend that jet radiation is likely from jet for LERG and from disk/corona for HERG. However, their sample is only 8 sources based on Fermi/LAT 1st catalog, and more sample RGs are needed to obtain a whole picture. In the context of this circumstance, we used the sample of 4FGL-DR2 catalog to increase the number of sample RGs. We investigated an origin of X-ray emission of each RG using FR classification and HERG/LERG classification [2] [3] [4], and report the result in this paper.

\section{Data}

In this paper, we use radio galaxy listed in 4FGL-DR2 catalog. This catalog has 61 objects classed as RDG, rdg, agn, css, and ssrq, and we chose 21 objects observed by Swift/XRT in X-ray. In this study, we use only PC mode data.

\section{X-ray spectral analysis}

Reduction of X-ray data is performed by using HeaSoft version 6.19. X-ray spectra and response files are created by xselect, grppha, and xrtmkarf. We extracted a source spectrum within 15 arcsec from the circle region centered on each RG, and a background spectrum at 105-135 arcsec from each RG. Source spectra were rebinned so as to contain 20 photon accounts in each bin when we apply $\chi^{2}$ statistics in the spectral fitting, of 1 photon count in each bin when we apply $\mathrm{C}$-statistics in the fitting. Spectral fitting is performed with xspec version 12.9.0. We fittied each of spectra in the energy range of $0.5-10.0 \mathrm{keV}$. For almost all of galaxies single-power-law model with 
Galactic absorption (phabs*pegp). The apec model is added (phabs (pegp+apec)) for galaxies where a thermal emission is seen in the soft X-ray band (3C111 and NGC1316). Spectral fitting is performed for each observation of our sample galaxies.

In this study, we obtained X-ray flux, X-ray photon index, and Hardness ratio for each observation. Hardness ratio is defined as Hardness ratio $=(\mathrm{H}-\mathrm{S}) /(\mathrm{H}+\mathrm{S})$ that is referred S.D.Connolly et al. 2016[5], where " $\mathrm{H}$ " is a count rate in the hard band of $2.0-10.0 \mathrm{keV}$, and " $\mathrm{S}$ " is a count rate in the soft band of 0.5-2.0 keV. In addition, we obtained an average of photon index and flux over all observations of each RG. Figure 1 shows the relation between hardness ratio and hard count rate of NGC 1218 which is one of the sample RGs. With the increase of hard count rate, hardness ratio shows higher value and thus this figure shows harder-when-brighter behavior. Also, almost all the sample RGs show this trend.

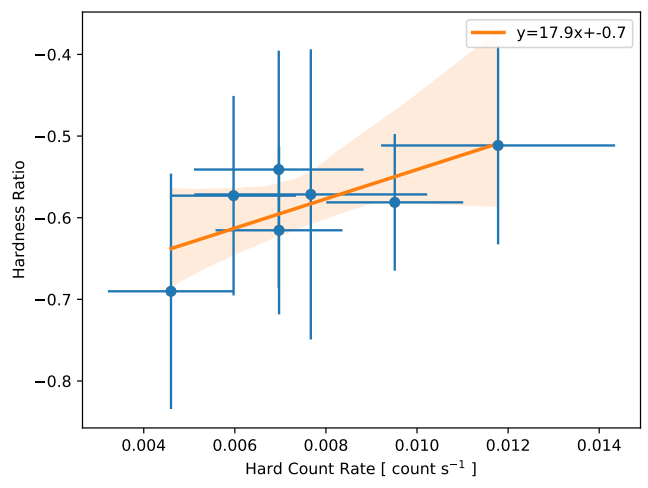

Figure 1: the relation between hardness ratio and hard count rate of NGC 1218

\section{Result}

\subsection{Time variability of X-ray spectra}

Figure 2 shows the harder-when-brighter slope against the X-ray to gamma-ray flux rate for our sample RGs. In this figure, it is apparent that the objects which have a low flux ratio around 0.3 and a small slope around 0.0 are concentrated. Hereafter we call objects at the left side of this figure as group A and the others as group B. First, the group A shows a relatively low gamma-ray flux and a weak harder-when-brighter trend, therefore, probably jet is weak in X-ray and it is inferred that disk/corona emission is observed mainly in X-ray. Second, the group B shows a relatively high gamma-ray flux and a strong harder-when-brighter trend. Therefore, jet radiation in X-ray is inferred.

\subsection{Relation between X-ray photon index and Gamma-ray photon index}

Figure 3 shows the relation between X-ray and gamma-ray photon index. We divide RGs into two groups; one group has a gamma-ray photon index of $<2.0$ and X-ray photon index of $>2.0$, and the other group has a gamma-ray photon index of $>2.0$ and X-ray photon index of $<2.0$. Therefore, the former group shows a hard spectra in gamma-ray and a soft spectra in X-ray and the latter group 


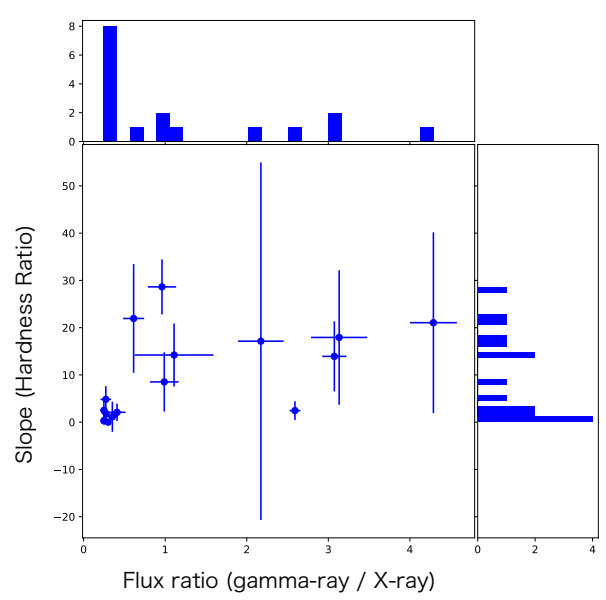

Figure 2: This figure shows the relation between flux ratio which is gamma-ray flux over X-ray flux and slope of hardness ratio, and shows histogram of these axis.

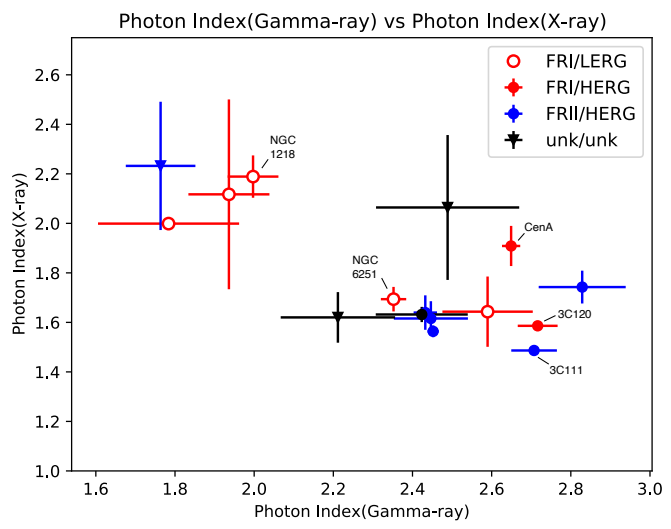

Figure 3: This figure shows the relation between X-ray photon index and gamma-ray photon index

shows a soft spectra in gamma-ray and a hard spectra in X-ray. In this study, former group is called as $\alpha$ group and latter group is called as $\beta$ group.

Figure 4 shows a SED trend of these two groups. $\alpha$ group and $\beta$ group correspond to left figure and right figure, respectively. Each figure show jet and disk/corona emission components. Group $\alpha$ shows a jet SED peaking at higher energy, due to higher energy of accelerated particles in the jet, while group $\beta$ shows a jet SED peaking at lower energy due to lower energy of accelerated particles. For disk/corona emission, when the accretion rate dotm is higher, the luminosity is proportional to $\dot{m}$. When $\dot{m}$ is lower, the luminosity is proportional to $\dot{m}^{2}$. Therefore, for FR-I/LERG(group $\alpha$ ) have a lower flux of disk/corona emission and thus jet emission is dominant in X-ray. For FR-II/HERG, disk/corona emission is bright, and thus significantly contribute to the X-ray band.

\subsection{Accretion Rate Using X-ray Luminosity}

Figure 5 shows a relation between a X-ray photon index and an Eddington luminosity ratio. In fig.4, FR-II/HERG objects shows relatively high Eddington ratio and FR-I/LERG objects show 


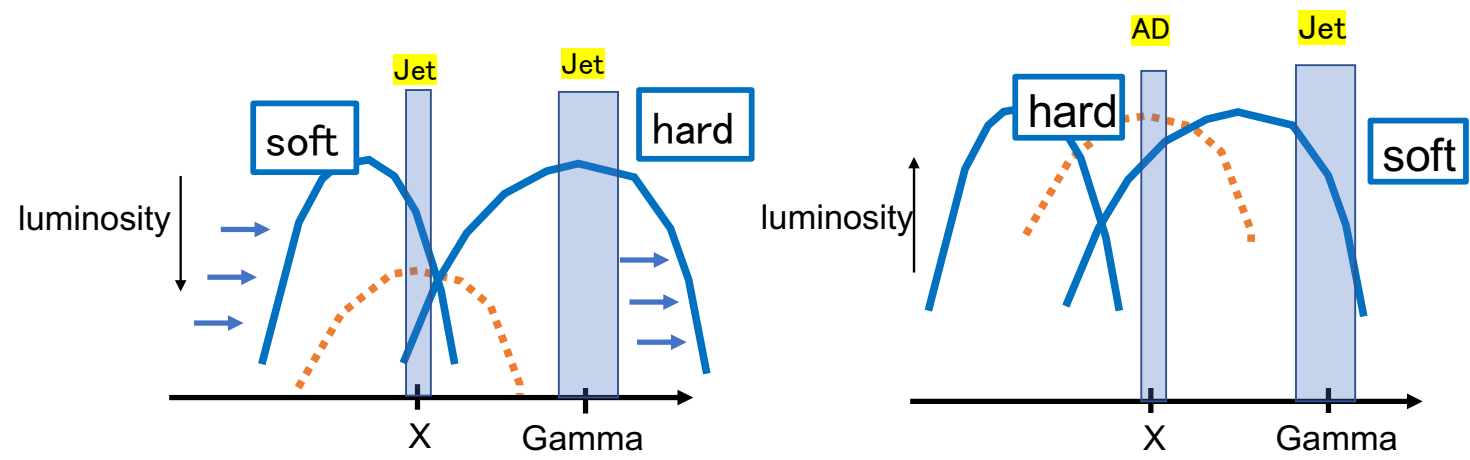

Figure 4: Simple Spectral Energy Distribution. The blue region shows observable energy regions. These are Swift/XRT in X-ray and Fermi/LAT in gamma-ray.

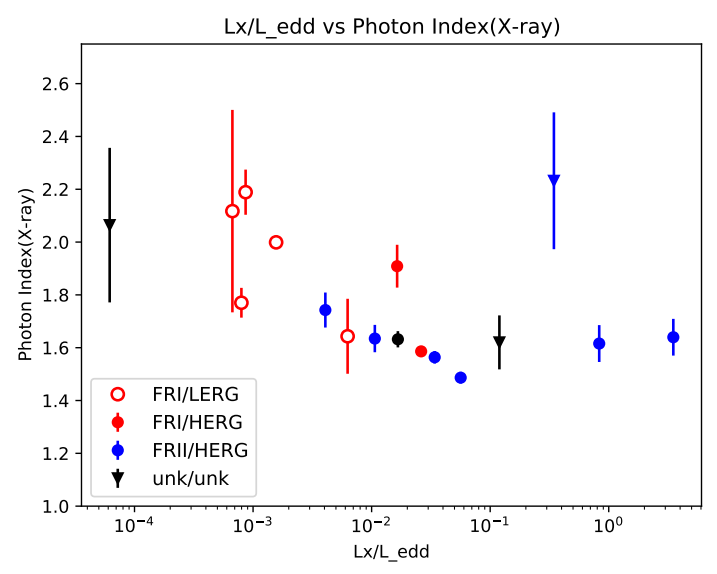

Figure 5: Relation between accretion rate and X-ray photon index

relatively low ratios, and this trend is the same as previous studies. FR-I/HERG objects show an intermediate ratio between the FR-II/HERG objects and the FR-I/LERG objects.

\section{Discussion and Conclusion}

\section{6. conclusion}

Up to this section, we reported several results to investigate the origin of X-ray radiation. Table 1 summarizes these results of each objects and FR and HERG/LERG classifications. We set a boundary Eddington ratio as 0.01, refering to the previous numerical simulation. Also, these classifications are refer to previous work[6].

Using these information on table 1, it turned out that objects are classified into 3 types. The first class "jet dominant type" which shows a strong harder-when-brighter behavior, a soft X-ray spectrum and a hard gamma-ray spectrum. The second is "Accretion disk dominant type" which shows a weak harder-when-brighter behavior, a hard X-ray spectrum and a soft gamma-ray spectrum. The third class is "jet and AD mixed type" which have intermediate properties. Also, it is confirmed 
that most of "jet dominant type" objects and "AD dominant type" objects correspond to the objects which are classified as a low or high accretion rate objects, based on the Eddington ratio and optical properties.

\begin{tabular}{ccccc}
\hline $\begin{array}{c}\text { Time variability } \\
\text { (Fig.1) }\end{array}$ & $\begin{array}{c}\text { Relation between } \\
\text { photon-indexes (Fig. 2) }\end{array}$ & $\begin{array}{c}L_{X} / L_{E d d} \\
\text { (Fig.4) }\end{array}$ & $\begin{array}{c}\text { Radio and Optical } \\
\text { classifications }\end{array}$ & Number \\
\hline \hline Jet & Jet & $<0.01$ & FR-I / LERG & 2 \\
& & $>0.01$ & FR-II / unk & 1 \\
\hline disk & disk and/or jet & $>0.01$ & FR-II / HERG & 3 \\
& & $>0.01$ & FR-I / HERG & 2 \\
& & $<0.01$ & FR-II / HERG & 1 \\
\hline jet & disk and/or jet & $<0.01$ & FR-I / LERG & 3 \\
& & $<0.01$ & unk / unk & 1 \\
& & $>0.01$ & FR-II / HERG & 3 \\
& & $>0.01$ & unk / unk & 1 \\
\hline \hline disk & jet & $<0.01$ & FR-I / LERG & 1 \\
\hline \hline
\end{tabular}

Table 1: conclution

\section{References}

[1] Yasushi Fukazawa, Justic Finke, Lukasz Stawarz, Yasuyuki Tanaka, Ryosuke Itoh, and Shin'ya Tokuda, 2014, arXiv:1410.2733

[2] B.L.Fanaroff and J.M.Riley, Monthly Notices of the Royal Astronomical Society, Vol. 167, p. 31P-36P (1974)

[3] S.Buttiglione, A.Capetti, A.Celotti, D.J.Axon, M.Chiaberge, F.D.Macchetto, and W.B.Sparks, 2010, A\&A Volume 509, A6

[4] D.Macconi, E.Torresi, P.Grandi, B.Boccardi and C.Vignali, Monthly Notices of the Royal Astronomical Society, Volume 493, Issue 3, April 2020, Pages 4355-4366

[5] S.D.Connolly, I.M.McHardy, C.J.Skipper and D.Emmanoulopoulos, Monthly Notices of the Royal Astronomical Society, Volume 459, Issue 4, 11 July 2016, Pages 3963-3985

[6] Ken OHSUGA, Shin MINESHIGE, Masao MORI, and Yoshiaki KATO, Publications of the Astronomical Society of Japan, Volume 61, Issue 3, 25 June 2009, Pages L7-L11 